\title{
Coulomb Collisions as a Candidate for Temperature Anisotropy Constraints in the Solar Wind
}

\author{
S. Vafin ${ }^{1}$ (D), M. Riazantseva ${ }^{2}$ (iD, and M. Pohl ${ }^{1,3}$ (1) \\ ${ }^{1}$ Institute of Physics and Astronomy, University of Potsdam, D-14476 Potsdam-Golm, Germany \\ ${ }^{2}$ Space Research Institute, Russian Academy of Sciences, Moscow, Russia \\ ${ }^{3}$ DESY, Platanenallee 6, D-15738 Zeuthen, Germany \\ Received 2018 November 9; revised 2018 December 20; accepted 2018 December 28; published 2019 January 22
}

\begin{abstract}
Many solar wind observations at 1 au indicate that the proton (as well as electron) temperature anisotropy is limited. The data distribution in the $\left(A_{a}, \beta_{a, \|}\right)$-plane have a rhombic-shaped form around $\beta_{a, \|} \sim 1$. The boundaries of the temperature anisotropy at $\beta_{a, \|}>1$ can be well explained by the threshold conditions of the mirror (whistler) and oblique proton (electron) firehose instabilities in a bi-Maxwellian plasma, whereas the physical mechanism of the similar restriction at $\beta_{a, \|}<1$ is still under debate. One possible option is Coulomb collisions, which we revisit in the current work. We derive the relaxation rate $\nu_{a a}^{A}$ of the temperature anisotropy in a bi-Maxwellian plasma that we then study analytically and by observed proton data from WIND. We found that $\nu_{p p}^{A}$ increases toward small $\beta_{p, \|}<1$. We matched the data distribution in the $\left(A_{p}, \beta_{p, \|}\right)$-plane with the constant contour $\nu_{p p}^{A}=2.8 \cdot 10^{-6} \mathrm{~s}-1$, corresponding to the minimum value for collisions to play a role. This contour fits rather well the left boundary of the rhombic-shaped data distribution in the $\left(A_{p}, \beta_{p, \|}\right)$-plane. Thus, Coulomb collisions are an interesting candidate for explaining the limitations of the temperature anisotropy in the solar wind with small $\beta_{a, \|}<1$ at 1 au.
\end{abstract}

Key words: instabilities - plasmas - scattering - solar wind - Sun: heliosphere

\section{Introduction}

Despite being the most well-investigated astrophysical plasma, the solar wind still offers many unsolved problems. One of them refers to the temperature anisotropy of the solar wind particles, $A_{a}=T_{a, \perp} / T_{a, \|}$, where $T_{a, \perp}$ and $T_{a, \|}$ are, respectively, the perpendicular and parallel with the respect to the ambient magnetic field temperatures of plasma species a $(a=p, e)$. Various observations demonstrate that the temperature anisotropy of both protons and electrons is strongly limited, accordingly, by $0.1 \lesssim A_{p} \lesssim 10$ and $0.5 \lesssim A_{e} \lesssim 2$ (Hellinger et al. 2006; Matteini et al. 2007; Štverák et al. 2008; Bale et al. 2009; Maruca et al. 2011). However, such a behavior cannot be described by the simple adiabatic expansion of the solar wind predicting much larger temperature anisotropies (Griffel \& Davis 1969; Phillips \& Gosling 1990). Thus, other physical processes must be in play.

One alternative explanation was proposed by Servidio et al. (2014), who used hybrid Vlasov-Maxwell simulations to study the evolution of the solar wind plasma. Their results show that starting with an isotropic turbulent Maxwellian plasma, turbulent processes are able to build up the temperature anisotropy themselves. Moreover, they predict a distribution of the temperature anisotropy in the $\left(\beta_{p, \|}, A_{p}\right)$-plane that is very similar to the observed data (Bale et al. 2009), but, nevertheless, the limitations of the temperature anisotropy in the whole parameter range are not yet fully understood. Here,

$$
\beta_{p, \|}=8 \pi n_{p} T_{p, \|} / B^{2},
$$

where $n_{p}$ is the proton density and $B$ the ambient magnetic field strength.

Different from Servidio et al. (2014), another group of studies invokes plasma instabilities that can be induced in a biMaxwellian plasma, setting limitations on the temperature anisotropy (Stix 1962; Hellinger et al. 2006; Štverák et al. 2008; Hellinger \& Trávníček 2014). However, the existing instability conditions can explain the temperature anisotropy trends only for $\beta_{a, \|}>1$, yet they fail to explain the observations at $\beta_{a, \|}<1$. It was found later that the presence of a relative drift in the plasma can additionally destabilize it and dramatically modify the instability conditions at small $\beta_{a, \|}$ (Ibscher et al. 2013; Ibscher \& Schlickeiser 2013, 2014; Chen et al. 2016). However, it requires a fairly large drift velocity and a high density of the drifting component, which are rather rare in the solar wind at 1 au (Vafin et al. 2018).

Thus, there is still no satisfactory explanation of the temperature anisotropy constraints in the solar wind with small $\beta_{a, \|}<1$. Apart from the abovementioned processes, one more mechanism restricting the temperature anisotropy is Coulomb collisions. Particle collisions play an important role in the solar wind. They are responsible, for instance, for the regulation of the electron heat flux and for the proton heating through alpha particles (Salem et al. 2003; Hellinger 2016). In addition to this, the observations show an increase of the collisional age toward small (as well as large) $\beta_{p, \|}$ (Bale et al. 2009). Although Bale et al. (2009) considered the collision frequency of a thermal plasma to calculate the collisional age of the solar wind, but their work does not analyze in detail how collisions affect the observed boundaries of the temperature anisotropy in the solar wind at $1 \mathrm{au}$. In contrast, in the current study we utilize the collision frequency of a bi-Maxwellian plasma to derive the relaxation rate of the temperature anisotropy. The inverse of this relaxation rate describes the characteristic time during which the temperature anisotropy changes due to particle collisions; therefore, this rate can be used as a qualitative measure of the effect of collisions on the temperature anisotropy alternatively to the collisional age. We demonstrate that the contour lines of this relaxation rate can explain the observed limits of the solar wind temperature anisotropy at $\beta_{a, \|} \ll 1$ and, furthermore, that they can also 


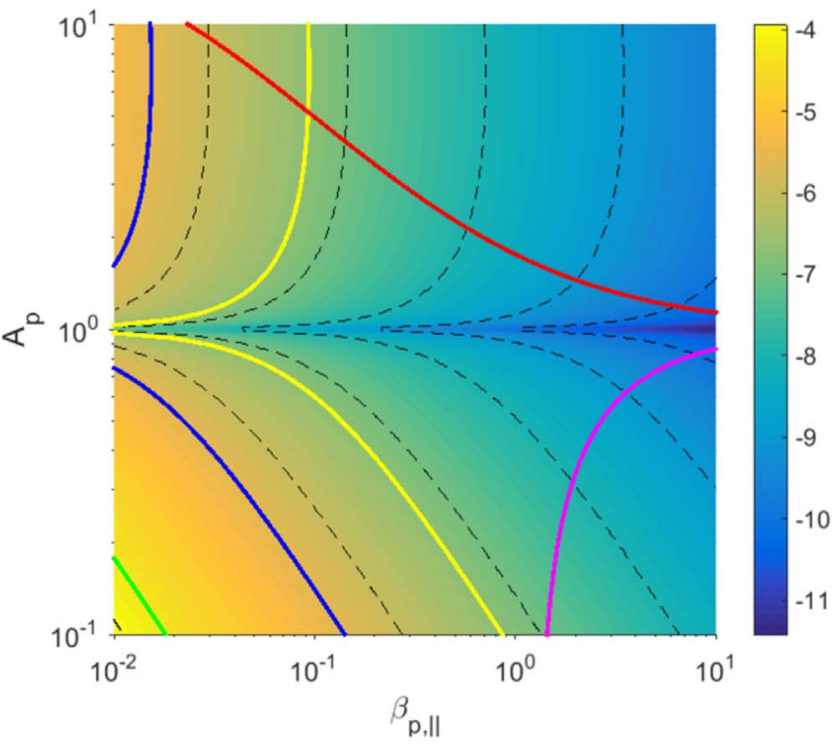

(a)

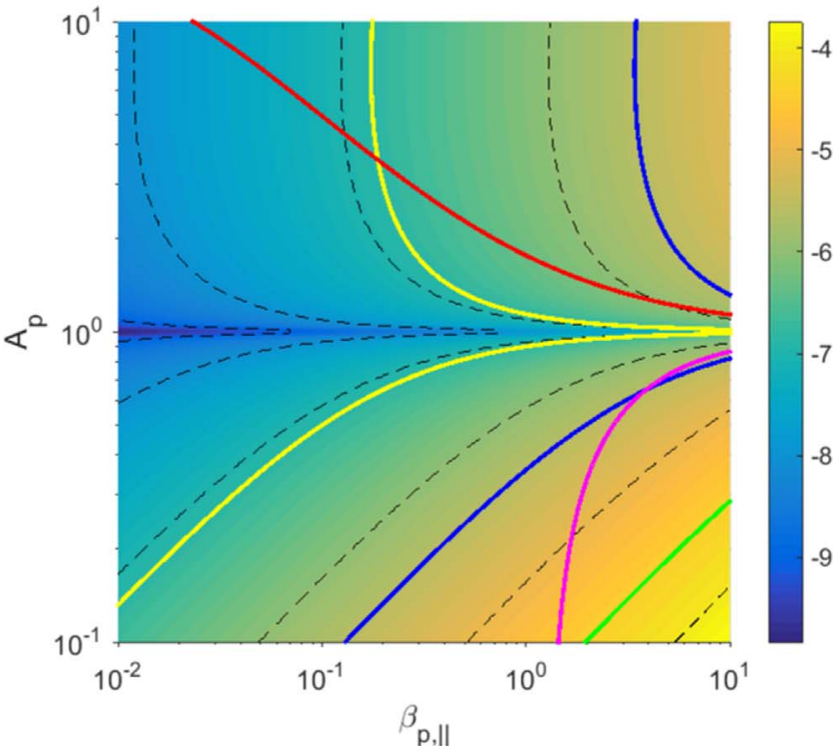

(b)

Figure 1. $\log _{10}$ of the proton-proton collision frequency $\nu_{p p}^{A}\left(\mathrm{~s}^{-1}\right), B=$ $10^{-4}$ G. Panel (a): $n=$ const $=3.4 \mathrm{~cm}^{-3}, 0.7<T_{p, \|}<700 \mathrm{eV}$. Panel (b): $T_{p, \|}=$ const $=10 \mathrm{eV}, 0.2<n<200 \mathrm{~cm}^{-3}$. The dashed lines illustrate the contours of constant $\nu_{a a}^{A}$ values. The green, blue, and yellow curves demonstrate the critical value $\nu_{a a}^{A}=2.6 \cdot 10^{-6} \mathrm{~s}^{-1}$ (Equation (12)) for $n=\mathrm{const}=1,3.4$, and $10 \mathrm{~cm}^{-3}$ in panel (a) and for $T_{p, \|}=$ const $=30,10$, and $3 \mathrm{eV}$ in panel (b), respectively. The red and magenta lines demonstrate the threshold conditions of mirror (Equation (14)) and oblique proton firehose (Equation (15)) instabilities, accordingly.

have an effect at $\beta_{a, \|} \sim 1$ providing stronger limitations than the instability thresholds.

The rest of the Letter is organized as follows. In Section 2, we briefly explain and illustrate our theoretical framework. Section 3 provides comparison with observations. The final discussion is presented in Section 4.

\section{Theoretical Model}

We will model the solar wind protons $(a=p)$ and electrons $(a=e)$ with a non-relativistic bi-Maxwellian distribution function

$$
f(\boldsymbol{r}, \boldsymbol{v})=\frac{n_{a}}{\pi^{3 / 2} u_{a, \|} u_{a, \perp}^{2}} \exp \left(-\frac{v_{\perp}^{2}}{u_{a, \perp}^{2}}-\frac{v_{\|}^{2}}{u_{a, \|}^{2}}\right),
$$

where $n_{a}$ is the number density of species $a$ and $u_{a, \perp}=\sqrt{2 T_{a, \perp} / m_{a}}$ and $u_{a, \|}=\sqrt{2 T_{a, \|} / m_{a}}$ denote, respectively, the perpendicular and parallel thermal velocities. We will assume that the solar wind has a constant homogeneous number density, $n_{a}=n$. The evolution of the proton and electron temperatures due to Coulomb collisions can be described by (Hellinger \& Trávníček 2009)

$$
\begin{gathered}
\frac{d T_{a, \|}}{d t}=-\nu_{a a}^{\|} T_{a, \|} \operatorname{sgn}\left(1-A_{a}\right), \\
\frac{d T_{a, \perp}}{d t}=\nu_{a a}^{\perp} T_{a, \perp} \operatorname{sgn}\left(1-A_{a}\right),
\end{gathered}
$$

where

$$
\begin{aligned}
& \nu_{a a}^{\|}=\frac{16 \sqrt{\pi} e^{4} n L_{a a}\left|A_{a}-1\right|}{15 \sqrt{m_{a}}\left(k_{B} T_{a, \|}\right)^{3 / 2}} F\left(2, \frac{3}{2} ; \frac{7}{2} ; 1-A_{a}\right), \\
& \nu_{a a}^{\perp}=\frac{8 \sqrt{\pi} e^{4} n L_{a a}\left|A_{a}-1\right|}{15 \sqrt{m_{a}} A_{a}\left(k_{B} T_{a, \|}\right)^{3 / 2}} F\left(2, \frac{3}{2} ; \frac{7}{2} ; 1-A_{a}\right) .
\end{aligned}
$$

Here, $F(a, b ; c ; x)$ denote the Gauss hypergeometric function and $\operatorname{sgn}(x)=|x| / x$. In Equations (3)-(4), we take into account only collisions between the particles of the same species (e-e, $\mathrm{p}-\mathrm{p}$ ), as the interspecies collisions are much less efficient. Then, the Coulomb logarithm $L_{a a}$ is (Huba 2016)

$$
L_{e e}=23.5-\ln \left(\frac{n_{e}^{1 / 2}}{T_{e}^{5 / 4}}\right)-\left[10^{-5}+\frac{1}{16}\left(\ln T_{e}-2\right)^{2}\right]^{1 / 2}
$$

and

$$
L_{p p}=23-\ln \left[\frac{\left(2 n_{i}\right)^{1 / 2}}{T_{i}^{3 / 2}}\right]
$$

for $\mathrm{e}-\mathrm{e}$ and $\mathrm{p}-\mathrm{p}$ collisions, respectively. Adding Equations (3)-(4), it is straightforward to find

$$
\frac{d A_{a}}{d t}=-\frac{1+2 A_{a}}{2 T_{a, \|}} \frac{d T_{a, \|}}{d t}
$$

or equivalently

$$
\frac{d A_{a}}{d t}=\nu_{a a}^{A} A_{a} \operatorname{sgn}\left(1-A_{a}\right)
$$

where $\nu_{a a}^{A}=\left[1+1 /\left(2 A_{a}\right)\right] \nu_{a a}^{\|}$. Thus, $\nu_{a a}^{A}$ serves as the characteristic relaxation rate for the temperature anisotropy and, thereby, it is a proper quantitative measure of the effect of collisions on the temperature anisotropy (alternatively to the isotropization frequency $\nu_{a a}^{\|, \perp}$ ). Let us normalize the time $t$ to the traveling time of the solar wind $L / V_{\mathrm{SW}}$, where $L$ is the distance from the Sun and $V_{\mathrm{SW}}$ is the solar wind speed. Then

$$
\frac{d A_{a}}{d x}=\frac{\nu_{a a}^{A} L}{V_{\mathrm{SW}}} A_{a} \operatorname{sgn}\left(1-A_{a}\right),
$$

where $x=t V_{\mathrm{SW}} / L$. Thus, $\nu_{a a}^{A}$ should be compared to $V_{\mathrm{SW}} / L$. For collisions to have an effect at $1 \mathrm{au}$, this relaxation rate should be greater than the inverse of the solar wind traveling 
time from the Sun to the Earth,

$$
\nu_{a a}^{A}>V_{\mathrm{SW}} / L \approx 2.6 \cdot 10^{-6} \mathrm{~s}^{-1},
$$

where $L \approx 1$ au and $V_{\mathrm{SW}}=400 \mathrm{~km} \mathrm{~s}^{-1}$ is the average solar wind speed. Here, we implicitly assume that the temperature anisotropy of the solar wind is generated in the close vicinity of the Sun and then relaxes due to collisions on the way to the Earth. It is also useful to approximately express $\nu_{a a}^{A}$ in practical units for the average solar wind parameters at 1 au $\left(n=1 n_{1}\right.$ $\left.\mathrm{cm}^{-3}, T_{a, \|}=1 T_{a, \|, 1} \mathrm{eV}\right)$ :

$$
\begin{aligned}
& \nu_{a a}^{A}\left[s^{-1}\right]=C_{a a}\left|A_{a}-1\right|\left(1+\frac{1}{2 A_{a}}\right) \\
& \times F\left(2, \frac{3}{2} ; \frac{7}{2} ; 1-A_{a}\right) \frac{n_{1}}{T_{a, \|, 1}^{3 / 2}},
\end{aligned}
$$

where $C_{e e}=3.86 \cdot 10^{-5}$ and $C_{p p}=8.74 \cdot 10^{-7}$ for electron and proton collisions, respectively.

We aim to study $\nu_{a a}^{A}$ in the parameter space $\left(\beta_{a, \|}, A_{a}\right)$ that is also used in the data analysis. The plasma density and parallel temperature enter $\nu_{a a}^{A}$ independently. We expect that the solar wind exhibits a complex interplay of its parameters, and in Section 3 we will study $\nu_{a a}^{A}$ using observational data for the solar wind. In this section, a first glimpse of the behavior of $\nu_{a a}^{A}$ is afforded analytically by separately considering two cases, $n=$ const and $T_{a, \|}=$ const. As $\nu_{a a}^{A}$ does not explicitly depend on the strength of the magnetic field, we will assume it to be constant at a level that is typical for solar wind conditions, $B=10^{-4} \mathrm{G}$. This simplification helps us to understand the effect of different parameters on the temperature anisotropy limitations by the relaxation frequency $\nu_{a a}^{A}$.

Figure 1 illustrates the proton-proton collision frequency, $\nu_{p p}^{A}$, for constant $n$ (panel a) and $T_{a, \|}$ (panel b). The dashed black lines represent constant levels of $\nu_{p p}^{A}$. The solid green, blue, and yellow lines are contours corresponding to $\nu_{p p}^{A}=2.6 \cdot 10^{-6} \mathrm{~s}^{-1}$ for three choices of constant $n$ (panel a) and $T_{p, \|}$ (panel b). For comparison, the solid red and magenta lines stand for the threshold conditions of the mirror and oblique proton firehose instabilities, respectively. In comparison to the ion cyclotron mode and the parallel proton firehose mode, these instability conditions give a better description for the observed constraints of the solar wind temperature anisotropy and can be described, respectively, as (Hellinger et al. 2006; Bale et al. 2009)

$$
\begin{gathered}
A_{p}>A_{M}=1+\frac{0.77}{\left(\beta_{p, \|}+0.016\right)^{0.76}}, \\
A_{p}<A_{I F}=1-\frac{1.4}{\beta_{p, \|}+0.11} .
\end{gathered}
$$

The plasma is unstable at $A_{p}>A_{M}$ (above the red curve in Figure 1) and $A_{p}<A_{I F}$ (below the magenta curve in Figure 1).

Similar results for electron-electron collisions are presented in Figure 2. For electrons, the fastest instabilities are whistler waves and the oblique electron firehose modes whose threshold conditions read (Gary \& Wang 1996; Gary \& Nishimura 2003; Hellinger et al. 2014; Lazar et al. 2017)

$$
A_{e}>A_{W}=1+\frac{0.25}{\beta_{e, \|}^{0.5}},
$$

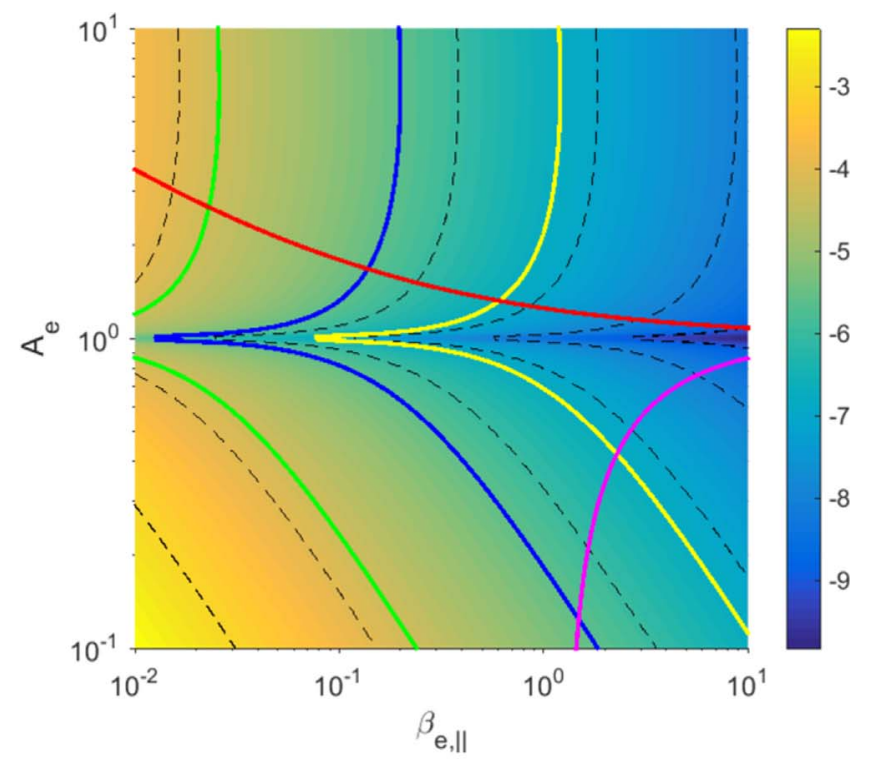

(a)

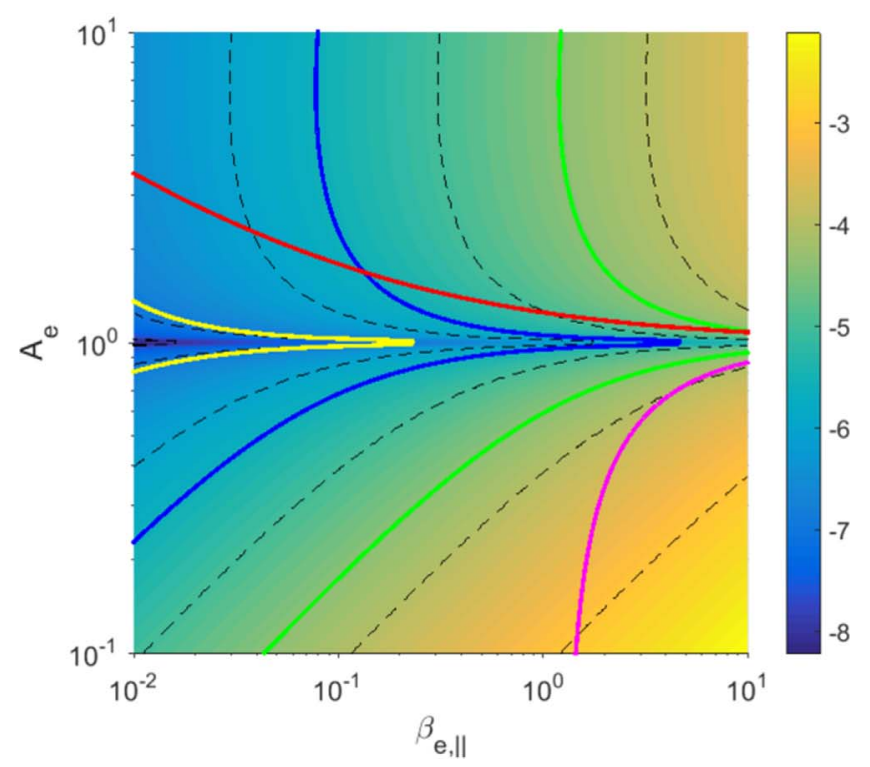

(b)

Figure 2. Same as Figure 1, but for electron-electron collisions. We used the same parameters as for protons in Figure 1. The red and magenta lines demonstrate the threshold conditions of whistler (Equation (16)) and oblique electron firehose (Equation (17)) instabilities, respectively.

$$
A_{e}<A_{E F}=1-\frac{1.29}{\beta_{e, \|}^{0.97}}
$$

The functions $A_{W}$ and $A_{E F}$ are illustrated in Figure 2 by the red and magenta line, respectively.

It should be noted that for Figures 1(a) and 2(a), we consider a maximum plasma temperature of $700 \mathrm{eV}$. Such a high solar wind temperature is never observed at $1 \mathrm{au}$, although it may be relevant for other regions close to the solar corona. Actually, the frequency $\nu_{a a}^{A}$ in Figures 1(a) and 2(a) becomes high enough at $\beta_{a, \|}<1$, where $T_{a, \|}<70 \mathrm{eV}$ and, thus, our main results on the collisional effects are well applicable in the range of solar wind parameters at $1 \mathrm{au}$.

It is well seen from Equation (5) that the relaxation rate, $\nu_{a a}^{A}$, is high at large plasma densities or small parallel temperatures. 
Figures $1-2$ show that at both $\beta_{a, \|} \ll 1$ and at $\beta_{a, \|} \gtrsim 1$ the scattering rate, $\nu_{a a}^{A}$, can exceed the critical value, above which it is relevant in the solar wind (marked by the blue line, Equation (12)).

One might be tempted to relate the blue contour (Equation (12), Figures 1(a) and 2(a)) to the observed limits of the solar wind temperature anisotropy in the range $\beta_{a, \|} \lesssim 0.1$ at 1 au (Štverák et al. 2008; Bale et al. 2009). Care must be exercised however, because the position of this contour depends on the plasma density. For a low-density plasma the contour shifts leftward to smaller $\beta_{a, \|}$ (especially for protons), where it does not agree well with the data.

The behavior of $\nu_{a a}^{A}$ in the intermediate range $0.1 \lesssim \beta_{a, \|} \lesssim 1$ is also of high interest. Figures 1(b) and 2(b) demonstrate that Coulomb collisions can restrict the temperature anisotropy even more than the relevant instabilities. For high-temperature protons (Figure 1(b)), the minimum required collision rate, marked by the yellow line, indicates stronger limitations on the temperature anisotropy than the oblique proton firehose and the mirror instability would impose. If the solar wind deviated from the isotropic state, its temperature anisotropy would be reduced by collisions before the solar wind becomes unstable to the oblique proton firehose or mirror instability. With increasing parallel proton temperature, the effect of collisions on the temperature anisotropy becomes negligible compared to that of plasma instabilities. For electrons (Figure 2(b)), the picture is similar, but even at relative large temperatures $\left(T_{e, \|}=30 \mathrm{eV}\right)$ collisions can dominate over the oblique electron firehose instability. This might explain why the measured distribution of the electron temperature anisotropy in the $\left(A_{e}, \beta_{e, \|}\right)$-plane does not fit well to the instability threshold of the oblique electron firehose mode (Štverák et al. 2008).

Thus, the behavior of the collision frequency, together with the instability thresholds, can qualitatively explain the rhombicshaped form of the solar wind data distribution in $\left(\beta_{a, \|}\right.$, $A_{a}$ )-plane (Bale et al. 2009).

\section{Comparison to Observations}

Here, we analyze the relaxation rate $\nu_{a a}^{A}$ and match it with measured data distribution in $\left(A_{a}, \beta_{a, \|}\right)$-plane for solar wind protons using observations from the WIND spacecraft at $1 \mathrm{au}$. Implication for solar wind electrons will be investigated in a separate paper. For the statistical analysis we use the measurement of plasma parameters (proton density, bulk velocity, and temperature) by the Solar Wind Electron (SWE) instrument (Ogilvie et al. 1995) based on two Faraday cups. The time resolution of plasma measurements is equal to 1.5 minutes. The interplanetary magnetic field parameters is measured by the Multi Frequency Instrument (MFI; Lepping et al. 1995) with better cadence but averaged to the same time resolution as the plasma parameters. The parallel and perpendicular components of proton temperature $\left(T_{p, \|}\right.$, $T_{p, \perp}$ ) were calculated by the average magnetic field direction using approach described in Kasper et al. (2002). We obtain long time series of WIND measurements in the ambient solar wind during the period from 1994 November 21 to 2017 December 31 by the Coordinated Data Analysis Web (CDAWEB) database. ${ }^{4}$ The total number of independent data points is equal to $8 \cdot 10^{6}$. The input measurements are processed by Hampel and median filters to detect and remove

\footnotetext{
https://cdaweb.sci.gsfc.nasa.gov
}

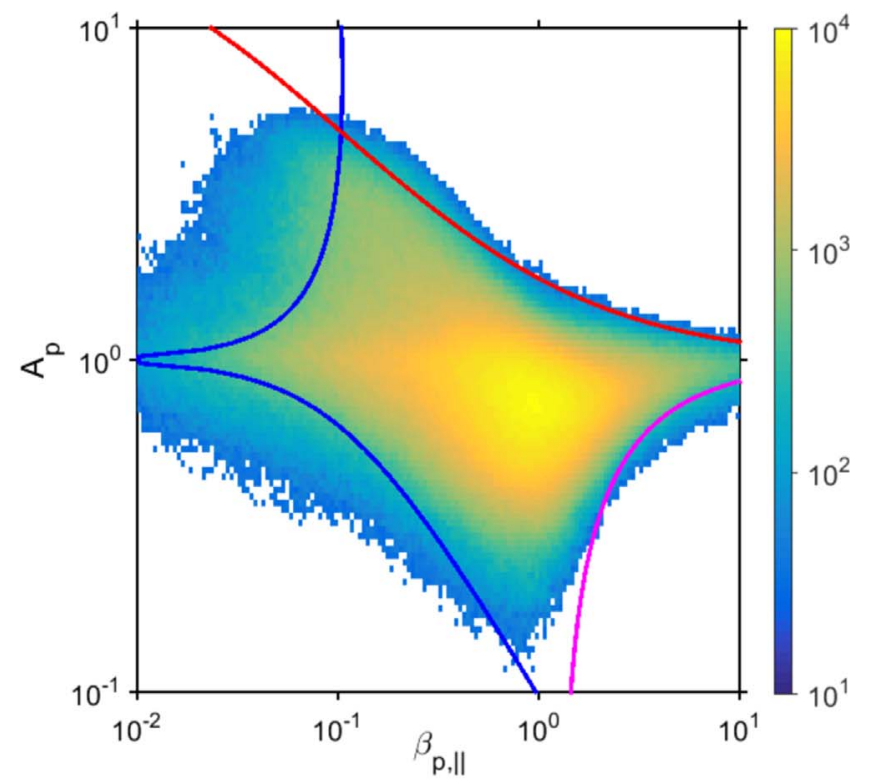

(a)

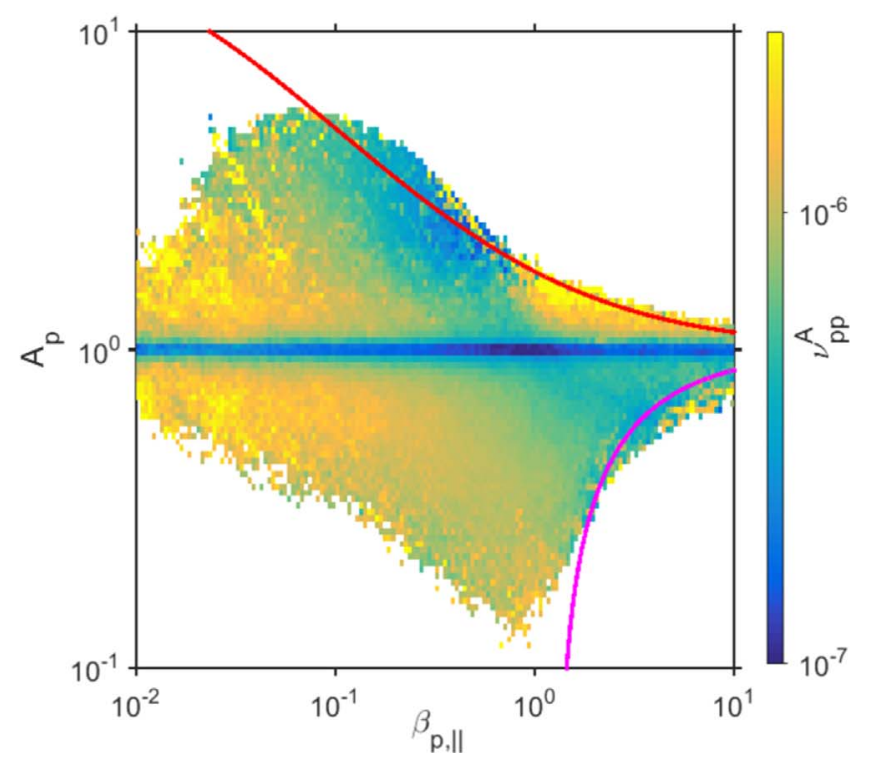

(b)

Figure 3. Upper panel: distribution of measurements $\left(A_{p}, \beta_{p, \|}\right)$ in the solar wind by WIND data set at 1 au. The blue line shows the contour $\nu_{p p}^{A}=2.8 \cdot 10^{-6} \mathrm{~s}^{-1}$ (Equation (18)) for $n=6 \mathrm{~cm}^{-3}, B=6 \cdot 10^{-5} \mathrm{G}, 0.1<T_{p, \|}<15 \mathrm{eV}$. Lower panel: proton-proton collision frequency $\nu_{p p}^{A}\left(s^{-1}\right)$. The horizontal blue region near $A_{p}=1$ in panel (b) appears because $\nu_{p p}^{A}$ approaches zero at $A_{p}=1$. The red and magenta lines demonstrate the threshold conditions of mirror (Equation (14)) and oblique proton firehose (Equation (15)) instabilities, respectively.

non-physical data outliers and reduce data noise. All data points are divided into logscale bins in $A_{p}$ and $\beta_{p, \|}$ space. Bins containing fewer than 30 data points are not shown at the subsequent figures. Figure 3(a) shows the distribution of proton temperature anisotropy $A_{p}$ against parallel proton plasma parameter $\beta_{p, \|}$, the color shows the number of points in each bin. This distribution is similar to those presented in Bale et al. (2009), but it is obtained by a longer data period (23 years of measurements). As discussed in recent papers, the distribution of the proton temperature anisotropy for $\beta_{p, \|} \geqslant 1$ is constrained by the threshold conditions of different plasma instabilities 
(Gary et al. 2001; Kasper et al. 2002; Bale et al. 2009), but the limitation of the temperature anisotropy for $\beta_{p, \|}<1$ has no clear explanation until now. Red and magenta lines on Figure 3(a) (as well as on Figure 3(b)) illustrate the threshold conditions of the mirror and oblique proton firehose instabilities, respectively. These threshold conditions can explain the core part of the observations rather well.

In the previous section we discussed the limitation of the temperature anisotropy due to Coulomb collisions. Figure 3(b) shows the relaxation rate $\nu_{p p}^{A}$ due to proton collisions computed from Equation (5) using experimental values of solar wind parameters and averaged in each bin of the distribution at the Figure 3(a). It is well seen from Figure 3(b) that the relaxation rate $\nu_{p p}^{A}$ increases toward small $\beta_{\|}<1$ in agreement with the analytic results for varied parallel temperatures (Figures 1(a) and 2(a)). We checked this and found that, indeed, $\nu_{p p}^{A}$ was calculated from the WIND data growth at $\beta_{p, \|}<1$ due to a decrease in the parallel proton temperature. At the same time, there is a slight increase of $\nu_{p p}^{A}$ for $\beta_{p, \|}>1$ and $A_{p}>1$, which is due to the density growth similar to Figures 1(b) and 2(b). Nevertheless, this region of the enhanced collision frequency almost coincides with the threshold condition of the mirror instability and, therefore, is rather unimportant. Thus, the effect of collisions for solar wind protons becomes significant mainly at small $\beta_{p, \|} \ll 1$.

The average solar wind speed for $\beta_{p, \|}<1$ in current data set is $420 \mathrm{~km} \mathrm{~s}^{-1}$. Then, the relaxation rate $\nu_{p p}^{A}$ should be above $2.8 \cdot 10^{-6} \mathrm{~s}^{-1}$.

$$
\nu_{p p}^{A}>2.8 \cdot 10^{-6} \mathrm{~s}^{-1}
$$

for protons to experience at least one collision. The most observed values of $\nu_{p p}^{A}$ in Figure 3(b) lie below this value. However, it is to be noted that the observed solar wind has already traveled the distance of $1 \mathrm{au}$ and may be already isotropized enough to reduce the relaxation rate $\nu_{p p}^{A}$. To address this issue, it is useful to match the theoretical curve corresponding to $\nu_{p p}^{A}=2.8 \cdot 10^{-6} \mathrm{~s}^{-1}$ with the data distribution in Figure 3(a). We mentioned above that collisions dominate at $\beta_{\|}$due to small parallel plasma temperatures. We use the average observed solar wind density, $n=6 \mathrm{~cm}^{-3}$, and the magnetic field, $B=6 \cdot 10^{-5} \mathrm{G}$, to find the contour level $\nu_{p p}^{A}=2.8 \cdot 10^{-6}$ for various $T_{p, \|}$ (similar to Figure $1(\mathrm{a})$ ). This contour is shown by the blue line in Figure 3(a). The parallel solar wind temperature for the blue curve changes in the range $0.1<T_{p, \|}<15 \mathrm{eV}$. Figure 3(a) demonstrates a good agreement between the contour $\nu_{p p}^{A}=2.8 \cdot 10^{-6} \mathrm{~s}^{-1}$ and the left boundary of the rhombic-shaped data distribution. Therefore, Coulomb collisions are an interesting candidate for explaining the limitations of the proton temperature anisotropy at small $\beta_{p, \|}<1$ in the solar wind at 1 au. Additionally, the fact that some observational points in Figure 3(a) lie on the left side from the blue curve indicates that solar wind protons could actually experience more than one collision.

\section{Summary and Discussion}

In the current work, we revisited the effect of Coulomb collisions on the solar wind temperature anisotropy. We derived the relaxation rate $\nu_{a a}^{A}$ of the temperature anisotropy in a bi-Maxwellian plasma. $\nu_{a a}^{A}$ describes the characteristic rate at which Coulomb collisions isotropize an initially anisotropic bi-Maxwellian plasma. This relaxation rate can be used alternatively to the collisional age (Bale et al. 2009) to quantify the effect of collisions on the temperature anisotropy of the solar wind.

We investigated the dependence of $\nu_{a a}^{A}$ on the plasma temperature and density analytically. We conclude that the contour lines of $\nu_{a a}^{A}$ at $\beta_{a, \|}<1$ in couple with the instability thresholds of mirror (whistler) and oblique proton (electron) firehose modes at $\beta_{a, \|}>1$ can potentially explain the full rhombic-shaped data distribution of solar wind temperature anisotropy in the $\left(A_{a}, \beta_{a, \|}\right)$-plane from WIND at $1 \mathrm{au}$. Furthermore, we found that the Coulomb collisions can be more restrictive than the instability thresholds at $\beta_{a, \|}>1$, which is especially true for solar wind electrons in qualitative agreement with the observations (Štverák et al. 2008).

We analyzed the relaxation rate $\nu_{a a}^{A}$ for solar wind protons using WIND data as described in Section 3. Our comparison demonstrates that the effect of collisions in this case is important at small $\beta_{p, \|}<1$, where $\nu_{p p}^{A}$ grows with decreasing $\beta_{\|}$in agreement with the analytic model. For the average solar wind parameters at $\beta_{p, \|}<1$, the relaxation rate $\nu_{p p}^{A}$ should be equal at least to $2.8 \cdot 10^{-6} \mathrm{~s}^{-1}$. In Figure 3(a), we matched the contour $\nu_{p p}^{A}=2.8 \cdot 10^{-6} \mathrm{~s}^{-1}$ (blue lines in Figure 3(a)) with the solar wind data distribution in $\left(A_{p}, \beta_{p, \|}\right)$-plane in couple with the instability thresholds (Equations (14)-(15)). We found that the contour $\nu_{p p}^{A}=2.8 \cdot 10^{-6} \mathrm{~s}^{-1}$ agrees well with the left boundary on the rhombic-shaped data distribution from WIND measurements. Thus, Coulomb collisions are an interesting candidate for explaining the limitations of the temperature anisotropy in the solar wind with small $\beta_{a, \|}<1$ at $1 \mathrm{au}$.

Here, we have considered the relaxation of the temperature anisotropy only due to particle-particle collisions. It should be noted, however, that the Coulomb collisions may be not the dominant scattering process. It was pointed out recently that the scattering between ion-acoustic waves and particles act faster than the Coulomb scattering in the solar wind at 1 au (Wilson et al. 2018). Accounting for the wave-particle interactions can put stronger constraints on the temperature anisotropy in the solar wind. Therefore, in a sense our results provide the lower limits on the temperature anisotropy. Moreover, in Equation (10), which describes the evolution of the temperature anisotropy, we did not consider the processes responsible for the deviation of the solar wind from the isotropic state. This circumstance is crucial for the establishment of the stationary distribution of the temperature anisotropy in the solar wind and could provide a better fitting for the observations. These effects should be in scope of future investigations.

We are grateful to the anonymous referee whose useful comments helped us to considerably improve the quality of our manuscript.

\section{ORCID iDs}

S. Vafin (1) https://orcid.org/0000-0003-2368-2801

M. Riazantseva (i) https://orcid.org/0000-0003-0501-2046

M. Pohl (i) https://orcid.org/0000-0001-7861-1707

\section{References}

Bale, S. D., Kasper, J. C., Howes, G. G., et al. 2009, PRL, 103, 211101 Chen, C. H. K., Matteini, L., Schekochihin, A. A., et al. 2016, ApJL, 825, L26 Gary, S. P., \& Nishimura, K. 2003, PhPl, 10, 3571 
Gary, S. P., Skoug, R. M., Steinberg, J. T., \& Smith, C. W. 2001, GeoRL, 28,2759

Gary, S. P., \& Wang, J. 1996, JGR, 101, 10749

Griffel, D. H., \& Davis, L. 1969, P\&SS, 17, 1009

Hellinger, P. 2016, ApJ, 825, 120

Hellinger, P., \& Trávníček, P. 2009, PhPl, 16, 054501

Hellinger, P., \& Trávníček, P. 2014, ApJL, 784, L15

Hellinger, P., Trávníček, P., \& Decyk, V. K. 2014, JGR, 119, 59

Hellinger, P., Trávníček, P., Kasper, J. C., \& Lazarus, A. J. 2006, GeoRL, 33, L09101

Huba, J. D. 2016, NRL Plasma Formulary (Washington, DC: Naval Research Laboratory)

Ibscher, D., Lazar, M., Michno, M. J., \& Schlikeiser, R. 2013, PhPl, 20, 012103

Ibscher, D., \& Schlickeiser, R. 2013, PhP1, 20, 042121
Ibscher, D., \& Schlickeiser, R. 2014, PhPl, 21, 022110

Kasper, J. C., Lazarus, A. J., \& Gary, S. P. 2002, GeoRL, 29, 1839

Lazar, M., Shaaban, S. M., Pierrard, V., Fichtner, H., \& Poedts, S. 2017, arXiv: 1704.05311

Lepping, R. P., Acũna, M. H., Burlaga, L. F., et al. 1995, SSRv, 71, 207

Maruca, B. A., Kasper, J. C., \& Bale, S. D. 2011, PhRvL, 107, 201101

Matteini, L., Landi, S., Hellinger, P., et al. 2007, GeoRL, 34, L20105

Ogilvie, K. W., Chornay, D. J., Fritzenreiter, R. J., et al. 1995, SSRv, 71, 55

Phillips, J. L., \& Gosling, J. T. 1990, JGR, 95, 4217

Salem, C., Hubert, D., Lacombe, C., et al. 2003, ApJ, 585, 1147

Servidio, S., Osman, K. T., Valentini, F., et al. 2014, ApJL, 781, L27

Stix, T. H. 1962, The Theory of Plasma Waves (New York: McGraw-Hill)

Vafin, S., Lazar, M., Fichtner, H., et al. 2018, A\&A, 613, A23

Štverák, S., Trávníček, P., Maksimovic, M., et al. 2008, JGR, 113, A03103

Wilson, L. B., III, Stevens, M. L., Kasper, J. C., et al. 2018, ApJS, 236, 41 